\title{
ERG6 and ERG2 are major targets conferring reduced susceptibility to amphotericin B in clinical Candida glabrata isolates in Kuwait
}

\author{
Khan Z ${ }^{\star 1}$, Ahmad S ${ }^{1}$, Joseph L11, Parker JE², Kelly SL², Asadzadeh $\mathrm{M}^{1}$
}

'Department of Microbiology, Faculty of Medicine, Kuwait University, Kuwait

${ }^{2}$ Institute of Life Science, Swansea University Medical School, Swansea, Wales, United Kingdom

\section{INTRODUCTION}

Candida glabrata is the second or third most commonly isolated yeast species causing candidemia and other invasive infections. $C$. glabrata is intrinsically less susceptible to azole antifungal drugs. Recent years have also witnessed increasing reports of breakthrough $C$. glabrata infections in patients receiving systemic echinocandin or polyene treatment. In this study, molecular mechanisms of reduced susceptibility (RS) to amphotericin B (AMB) were investigated in C. glabrata strains in Kuwait by sequence analyses of genes involved in ergosterol biosynthesis.

\section{METHODS}

(1) A total of 13 C. glabrata isolates (8 RS-AMB and 5 AMB-susceptible) were used. (2) Antifungal susceptibility test (AST) was done by Etest and by reference broth microdilution. (3) PCR-sequencing of three (ERG2, ERG6 and ERG11) genes was performed by using gene-specific primers. (4) The total cell sterol content was analyzed by gas chromatography-mass spectrometry. (5) Phylogenetic relationship among the isolates was investigated by multilocus sequence typing (MLST).

\section{RESULTS}

The AST data for AMB and other antifungal drugs by the reference broth microdilution method are presented in Table 1.

AMB-susceptible isolates contained only synonymous mutations in ERG2, ERG6 or ERG11 and total sterol content of 1 isolate was similar to reference strain (Table 2).

A nonsynonymous (AGA48AAA, R48K) ERG6 mutation was found in both RS-AMB and AMB-susceptible isolates.

$>$ Four RS-AMB isolates contained novel nonsense mutations at Trp286/Tyr192/Leu341 and two isolates contained nonsynonymous (V126F or C198F) mutation in ERG6 and their sterol content were consistent with ERG6 deficiency.

> Other RS-AMB $(n=2)$ isolates contained novel nonsynonymous (G119S or G122S) ERG2 mutation and their sterol content were consistent with ERG2 deficiency.

Isolate Kw861/13 also contained Y141H + L381M mutations while 7 RS-AMB isolates contained only synonymous mutations in ERG11.

Fingerprinting analysis revealed that all isolates with ERG6/ERG2/ERG11 mutations belonged to a unique genotype (Table 2).
Table 1. Source of isolation and MIC values of 13 C. glabrata isolates for AMB, FLC \& ANI by Etest and broth microdilution method

\begin{tabular}{|c|c|c|c|c|c|}
\hline \multirow{2}{*}{ Isolate } & Clinical & MIC $(\mu \mathrm{g} / \mathrm{ml})$ for & \multicolumn{3}{|c|}{$\begin{array}{c}\text { MIC values }(\mu \mathrm{g} / \mathrm{ml}) \text { by broth } \\
\text { microdilution method for }\end{array}$} \\
\hline no. & specimen & AMB by Etest & AMB & FLC & ANI \\
\hline ATCC90030 & N. A. & 0.094 & 0.5 & 4 & 0.016 \\
Kw600/09 & Wound swab & 0.032 & N. D. & N. D. & N. D. \\
Kw164/15 & Urine & 0.38 & 0.25 & 16 & 0.5 \\
Kw383/15 & Ascitic fluid & 0.19 & 0.5 & 2 & 0.016 \\
Kw590/15 & Sputum & 0.003 & 0.25 & 16 & 0.016 \\
Kw1421/16 & Bone & 0.25 & 0.5 & 1 & 0.031 \\
Kw844/10 & Urine & 1.5 & 1 & 2 & 0.031 \\
Kw861/13 & ET aspirate & 1 & 1 & $>64$ & 0.016 \\
Kw96/15 & Urine & 2 & 1 & 0.5 & 0.031 \\
Kw1856/15 & Urine & 1.5 & 1 & 0.25 & 0.016 \\
Kw2516/15 & Urine & 1.5 & 1 & 0.5 & 0.016 \\
Kw2813/15 & Urine & 4 & 1 & 0.25 & 0.016 \\
Kw3060/15 & Wound swab & 1.5 & 1 & 1 & 0.031 \\
Kw3357/15 & Urine & 2 & 1 & 2 & 0.031 \\
\hline
\end{tabular}

MIC, minimum inhibitory concentration; AMB, amphotericin B; FLC, fluconazole; ANI, anidulafungin; N. A., not available; N. D., not done

\section{CONCLUSIONS}

All isolates with ERG6/ERG2/ERG11 mutations belong to distinct strains.

Our data show that ERG6 and ERG2 are major targets conferring RS-AMB in clinical $C$. glabrata isolates in Kuwait.

Table 2. Susceptibility to AMB, mutations detected in ERG2, ERG6 and ERG11 genes, fingerprinting by ITS-ERG2-ERG6-ERG11 sequence-based profile in 13 C. glabrata isolates

\begin{tabular}{|c|c|c|c|c|c|}
\hline \multirow{2}{*}{$\begin{array}{l}\text { Isolate } \\
\text { no. }\end{array}$} & \multirow{2}{*}{$\begin{array}{l}\text { Susceptibility } \\
\text { to AMB }\end{array}$} & \multicolumn{3}{|c|}{ Non-synonymous mutations detected in } & \multirow{2}{*}{$\begin{array}{c}\text { ITS-ERG2-ERG6- } \\
\text { ERG11-based profile }\end{array}$} \\
\hline & & ERG2 & ERG6 & ERG11 & \\
\hline ATCC90030a & Susceptible & Reference $^{\mathrm{b}}$ & Reference $^{\mathrm{b}}$ & Reference $^{b}$ & Reference $^{\mathrm{b}}$ \\
\hline Kw600/09 & Susceptible & I207V & None & None & Unique \\
\hline Kw164/15 & Susceptible & I207V & None & None & Unique \\
\hline Kw383/15 & Susceptible & I207V & R48K & None & Unique \\
\hline Kw590/15 & Susceptible & I207V & R48K & None & Unique \\
\hline Kw1421/16 & Susceptible & I207V & None & None & Unique \\
\hline Kw844/10 & Resistant & $1207 V+G 122 S$ & None & None & Unique \\
\hline Kw861/13 & Resistant & I207V & $\Delta$ Nt. 1021, L341* & $\mathrm{Y} 141 \mathrm{H}+\mathrm{L} 381 \mathrm{M}$ & Unique \\
\hline Kw96/15 & Resistant & I207V & V126F & None & Unique \\
\hline Kw1856/15 & Resistant & I207V & W286* & None & Unique \\
\hline Kw2516/15 & Resistant & I207V & $\mathrm{R} 48 \mathrm{~K}+\mathrm{C} 198 \mathrm{~F}$ & None & Unique \\
\hline Kw2813/15 & Resistant & I207V & Y192* & None & Unique \\
\hline Kw3060/15 & Resistant & $1207 \mathrm{~V}+\mathrm{G} 119 \mathrm{~S}$ & None & None & Unique \\
\hline Kw3357/15 & Resistant & I207V & W286* & None & Unique \\
\hline
\end{tabular}

